\title{
Measurement of the Hybrid Photodiode Response - Fiber Optic Window
}

\author{
J. Freeman, D. Green and A. Ronzhin \\ Fermi National Accelerator Laboratory \\ P.O. Box 500, Batavia, Illinois 60510 \\ P. de Barbaro \\ University of Rochester
}

January 1999 


\section{Disclaimer}

This report was prepared as an account of work sponsored by an agency of the United States Government. Neither the United States Government nor any agency thereof, nor any of their employees, makes any warranty, express or implied, or assumes any legal liability or responsibility for the accuracy, completeness, or usefulness of any information, apparatus, product, or process disclosed, or represents that its use would not infringe privately owned rights. Reference herein to any specific commercial product, process, or service by trade name, trademark, manufacturer, or otherwise, does not necessarily constitute or imply its endorsement, recommendation, or favoring by the United States Government or any agency thereof. The views and opinions of authors expressed herein do not necessarily state or reflect those of the United States Government or any agency thereof.

\section{Distribution}

Approved for public release; further dissemination unlimited.

\section{Copyright Notification}

This manuscript has been authored by Universities Research Association, Inc. under contract No. DE-AC02-76CH0300O with the U.S. Department of Energy. The United States Government retains and the publisher, by accepting the article for publication, acknowledges that the United States Government retains a nonexclusive, paid-up, irrevocable, worldwide license to publish or reproduce the published form of this manuscript, or allow others to do so, for United States Government Purposes. 
Fermi National Accelerator Laboratory

\author{
FERMILAB-TM- 2064
}

\title{
Measurement of the Hybrid Photodiode Response - Fiber Optic Window
}

\author{
J.Freeman, D.Green, A.Ronzhin \\ Fermi National Accelerator Laboratory \\ P.O.Box 500, Batavia, Illinois 60504 \\ P. de Barbaro \\ University of Rochester \\ Rochester
}

January 1999 


\section{Measurement of the Hybrid Photodiode \\ Response - Fiber Optic Window}

\section{INTRODUCTION}

The hybrid photodiode (HPD [1]) will be used for the CMS HCAL readout [2]. A fiber-optic front window is used to reduce optical cross-talk between the pixels of the multi-pixel HPD's. A mismatch of numerical aperture between optical fibers carrying light to the HPD window and the fibers composing the fiber-optic front window of the HPD could lead to light loss. The light loss would appear as a reduced effective quantum efficiency of the device. The goal of this set of measurements was to see if there was in fact a reduction in the effective quantum efficiency of the HPD's.

\section{SETUP}

The setup is shown in fig.1. The scintillator plate (SCSN81, 240x160x4 $\mathrm{mm}^{3}$ ) with an embedded green fiber (Bicron, $0.93 \mathrm{~mm}$ diameter, polystyrene doped with Y11) was illuminated by a light emitting diode (LED, type NICHIA, Nichia Chemical Industries, NLPB 320 AS, emission maximum of $450 \mathrm{~nm}$ ). A green fiber with a full length of about $750 \mathrm{~mm}$ was inserted into the "sigma shaped" scintillator groove. The green fiber was optically connected to a clear fiber of the same diameter and length 1 meter. This clear fiber was used to measure the photocurrent of the photodetector under study. The length of the fiber outside the scintillator was 1.5 meters.

The photodetectors were:

1. Single channel HPD DEP with $25 \mathrm{~mm}$ diameter photocathode (tube type PP0350B).

2. Photomultiplier (PMT) Hamamatsu R5686 and R4598-01. The R5686 has a 53 mm diameter bialkali photocathode with a borosilicate glass window.

3. Pin diode (Hamamatsu S1722-02, $5 \mathrm{~mm}$ photocathode diameter).

A negative voltage was applied between the photocathode and the silicon surface to measure the HPD's photocurrent and between the photocathode and the first dynode for the PMT. In the latter case all electrodes of the photomultiplier (except for the photocathode) were connected together and shorted to ground through the Keithley multimeter (A, fig. 1). The pin diode was under reverse bias with the anode shorted through the ammeter. 


\section{MEASUREMENTS}

The photocurrent dependence on voltage was measured first (figs. 2, 3, 4, 5). The LED light used was adjusted to produce about $1 \mathrm{nA}$ of HPD photocurrent. The same amount of LED light was used later when working with the other photodetectors. A low photocurrent level was chosen in order to operate in a linear photocurrent range of the photodetectors. The illuminated fiber was installed on the photocathode perpendicular to it surface.

The noise current was measured without LED illumination and is also presented in figs. $2,3,4,5$.

An additional measurement was performed with the setup shown in fig. 6. The LED excited a plastic bar through a $1 \mathrm{~mm}$ diameter clear fiber of 1 meter length. The plastic bar was doped with Y11 light shifter. The size of the bar was $10 \times 15 \times 50 \mathrm{~mm}^{3}$ One of the $10 \times 15 \mathrm{~mm}^{2}$ surfaces was optically connected to the photocathodes. The fiber was inserted into the plastic bar from the side opposite to the photocathode. The Y11 bar was wrapped with several layers of white paper. The photocurrent was measured for the PMT and for the HPD with the same amount of light.

The photocathode nonuniformity over the Y11 area of illumination was less than $10 \%$. The value was measured by scanning the PC's with a $1 \mathrm{~mm}$ diameter fiber. The point of this measurement was to verify that the nonuniformity doesn't contribute significantly to the result.

\section{RESULTS}

The measured net photocurrent was defined to be the difference between the output current under LED illumination and without it (figs. 2, 3, 4, 5). There is a long plateau as a function of the potential between the photocathode and the collecting electrodes starting at a value of a few volts. The measured values of the photocurrent under the same light illumination are in good agreement with the photodetectors' quantum efficiency curves in the range of Y11 emission (fig. 7). The photocurrent of the PMT's and HPD is almost the same, as are the quantum efficiencics, which arc about 14-15\% at $495 \mathrm{~nm}$ (maximum of the Y11 emission spectrum). The pin diode photocurrent is approximatly 5 times higher than the HPD (or PMT) which is also consistent with the pin diode QE (fig. 7).

However the HPD's measured photocurrent is about 2.3 times less than the PMT's under the same light illumination level when the Y1l plastic bar is used (fig. 6).

\section{DISCUSSION}

No noticeable light losses were observed for the HPD with the fiber optic window when the light was transported to the photocathode by the optical fiber. Losses are thought to occur because of a mismatch between the incident angles of the light and the fiber optic numerical aperture. In other words, almost all the light coming 
out of the fiber is captured by the fiber optic glass window. The behavior is well matched to the CMS HCAL application.

The situation is different for the Y11 plastic bar (fig.6). A simple illustration of the phenomena is shown in fig.8. Only part of the incident light is captured by the fiber optic glass window in this case, leading to a reduced effective quantum efficiency.

\section{CONCLUSION}

The measured response of the HPD with the fiber optic glass window does not show significant light losses when coupled with CMS HCAL scintillator/fiber optic readout. 


\section{BIBLIOGRAPHY}

1. The Hybrid Photodiode tubes (HPD), Catalog of DEP DELFT INSTRUMENTS Co.

2. CMS. The Hadron Calorimeter Project. Technical Design

Report. CERN/LHCC 97-31, CMS TDR 2, 20 June 1997. 
Figures.

1. Setup to measure photocurrent.

$2,3,4,5$. The photodetector's photocurrent and noise vs applied voltage, 2 - photomultiplier (PMT) R5686, 3 - PMT

R4598-01, 4 - pin diode, 5 - HPD.

6. Setup for measurements with Y11 bar.

7. Quantum efficiencies of R5686, HPD, pindiode and emission spectrum of Y11.

8. Illustration of possible light losses for fiber optic faceplate. 


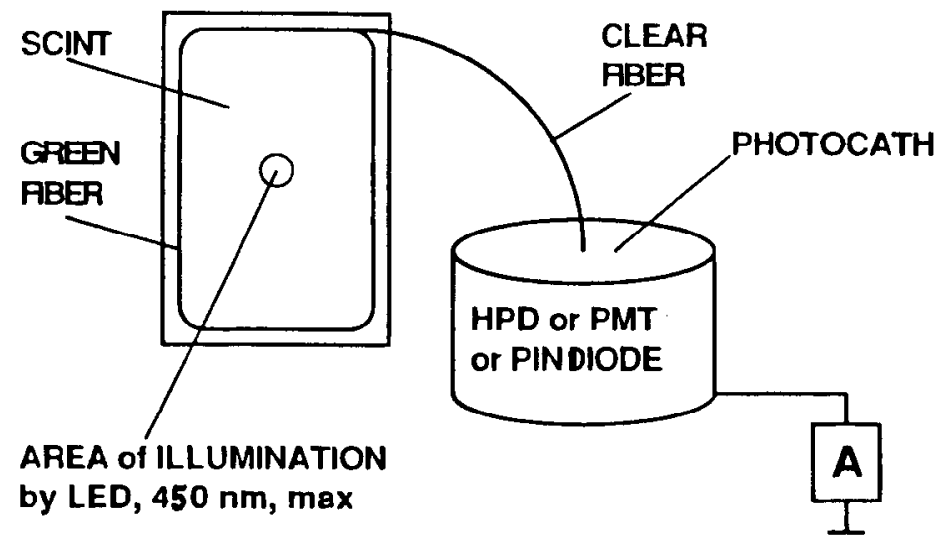

Fig.1

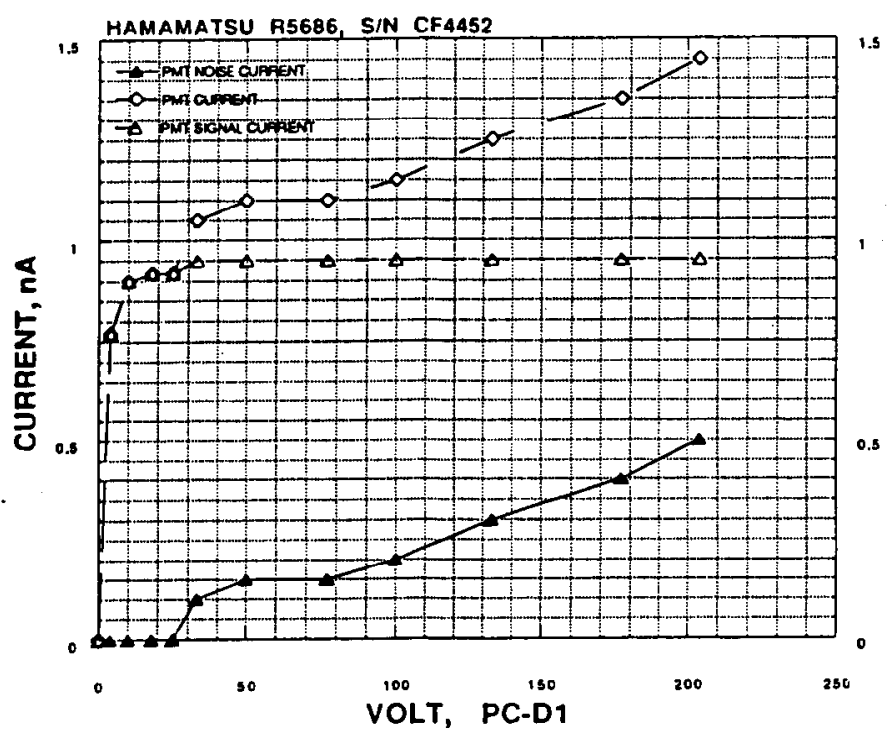

Fig.2

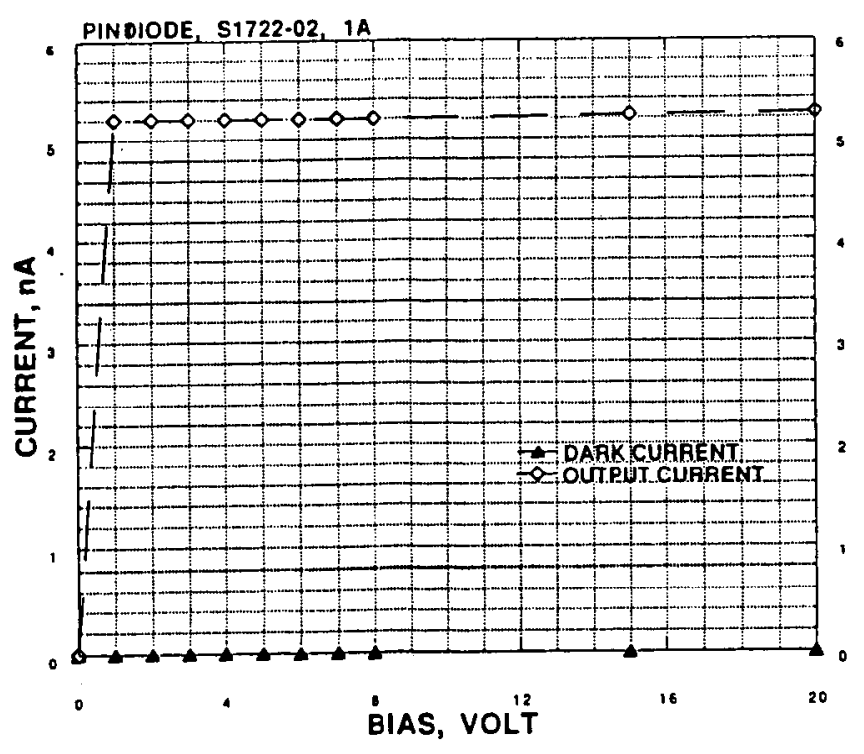

Fig.4

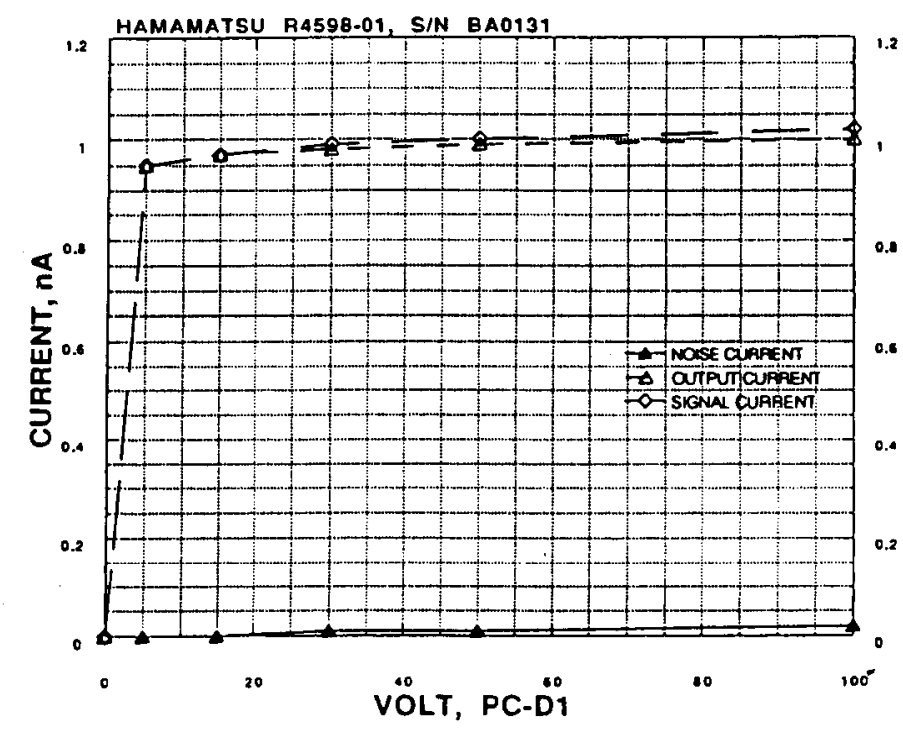

Fig.3

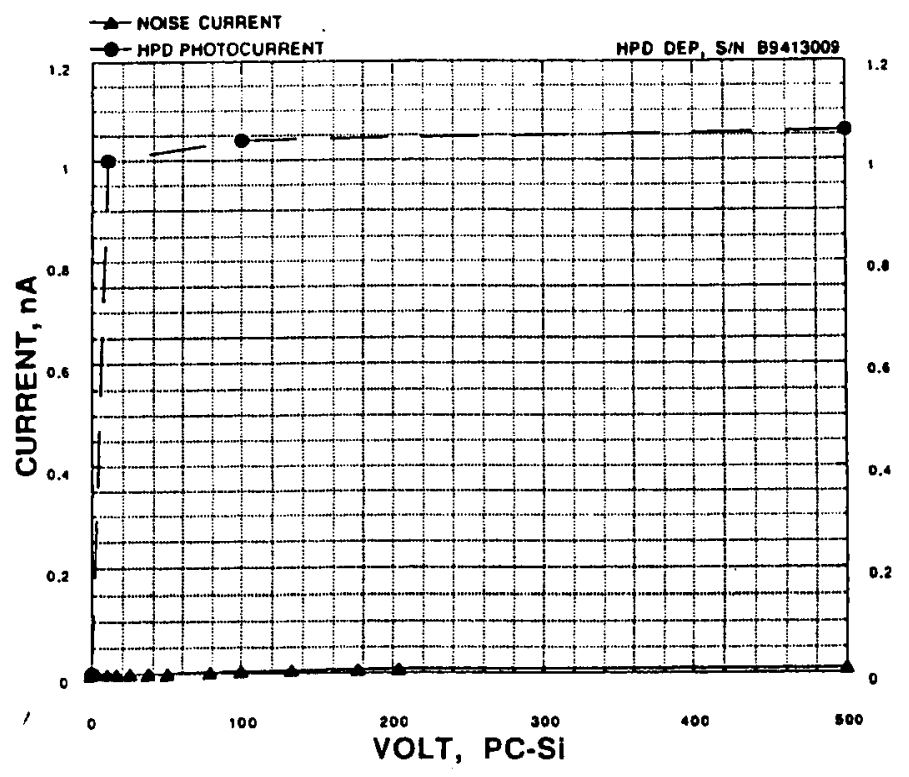

Fig.5 


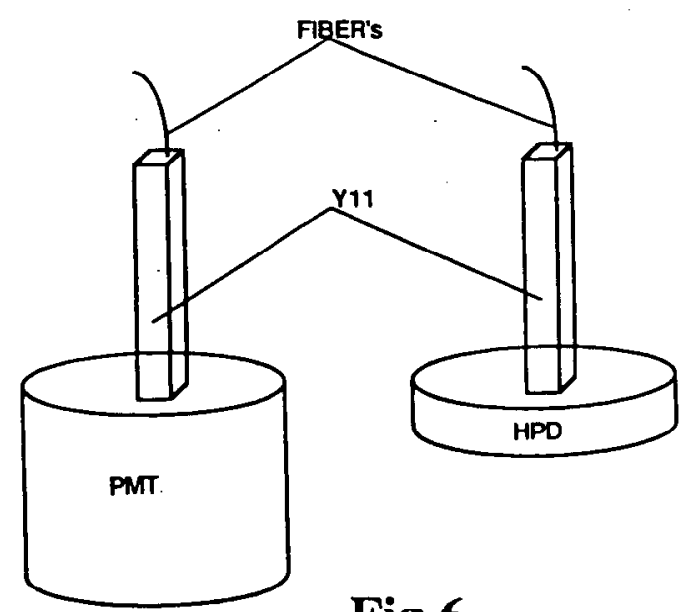

Fig.6

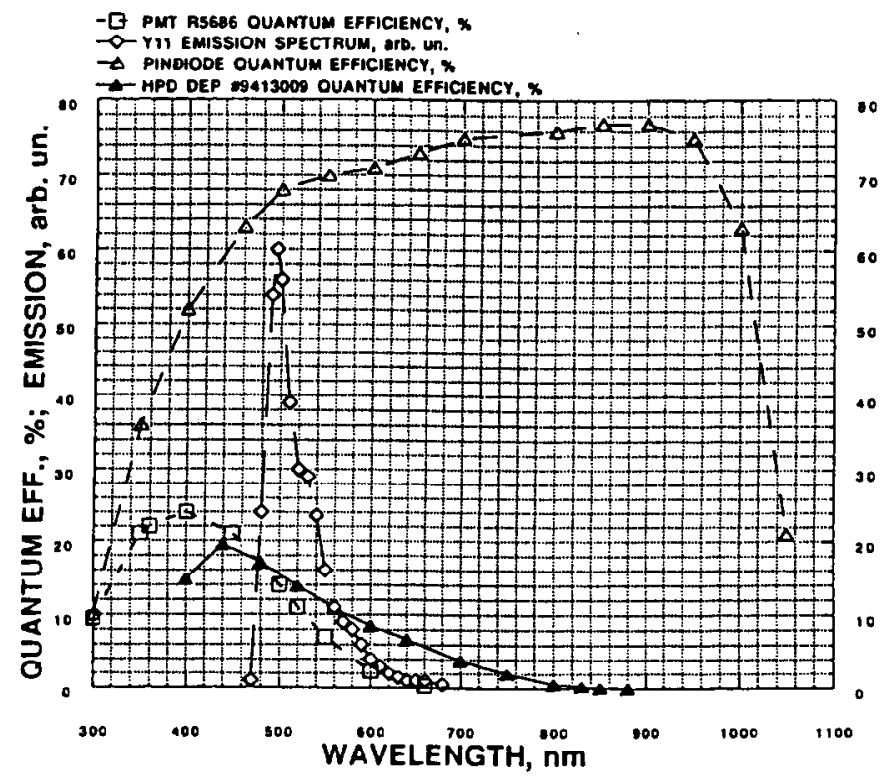

Fig.7

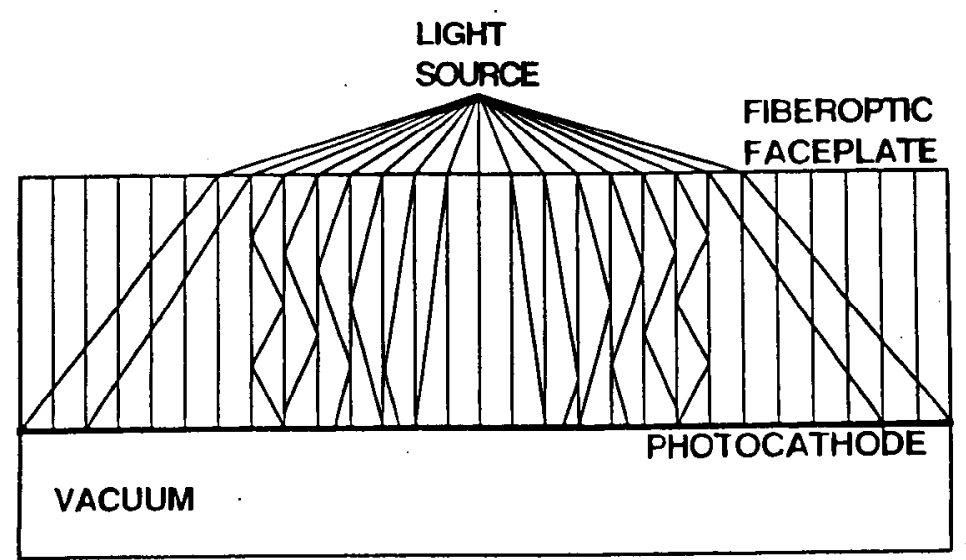

Fig.8 